\title{
Wind ringing of the ocean in presence of mesoscale eddies
}

\author{
P. Klein and G. Lapeyre \\ Laboraloire de Physique des Océans, Institut Français de Recherche pour l'Exploitation de la Mer, Plouzané France
}

\author{
W. G. Large \\ Climate and Global Dynamics Division, NCAR, Boulder, Colorado, USA
}

Received 16 April 2004; accepted 17 June 2004; published 7 August 2004.

[1] Recent findings have highlighted the impact of high frequency (HF) winds on the inertial motions and the consequences on the large-scale oceanic circulation [Wunsch and Ferrari, 2004]. Within this context the present study focuses on the role of intermediate scales related to oceanic mesoscale eddies. Results show that a turbulent eddy field does not affect the impact of $\mathrm{HF}$ winds on the inertial motions. However the eddies efficiently and permanently concentrate the wind-forced inertial motions in small-scale anticyclonic structures. This leads to a spatially heterogeneous vertical mixing strongly related to the eddy field properties. INDEX TERMS: 4504 Oceanography: Physical: Air/sea interactions (0312); 4544 Oceanography: Physical: Internal and inertial waves; 4520 Oceanography: Physical: Eddies and mesoscale procésses; 4572 Oceanography: Physical: Upper ocean processes; 4568 Oceanography: Physical: Turbulence, diffusion, and mixing processes. Citation: Klein, P., G. Lapeyre, and W. G. Large (2004), Wind ringing of the ocean in presence of mesoscale eddies, Geophys. Res. Lett., 31, L15306, doi:10.1029/2004GL020274.

\section{Introduction}

[2] Alford [2001] and Watanabe and Hibiya [2002] have pointed out the impact of high-frequency (HF) wind fluctuations (with periods smaller than 12 hours) for the magnitude of the mixed-layer inertial motions. Using Alford [2001] results, Wunsch and Ferrari [2004] estimate that these HF winds (through the inertial motions) have an effect on the large-scale oceanic circulation close to that due to the steady large-scale winds. These findings emphasize the necessity to quantify the contributions of intermediate scales and in particular the role of mesoscale eddies.

[3] The impact of $\mathrm{HF}$ winds on the inertial motions is explained by a resonance mechanism [Large and Crawford, 1995; Skillingstadt et al., 2000]. Initially, wind-forced inertial motions are mostly trapped within the oceanic mixed layer and have a frequency close to the Coriolis frequency $f$. The mixed-layer dynamics is therefore considered as a potential oscillator with a frequency close to $f$. If the windstress possesses some energy in the inertial frequency band with anticyclonic rotation in the same direction as the inertial motions, the mixed-layer oscillator will be excited, leading to a systematic increase of the kinetic energy and deepening of the mixed-layer. One important assumption made by all of the studies related to the ocean ringing, is the oceanic horizontal homogeneity

Copyright 2004 by the American Geophysical Union. 0094-8276/04/2004GL020274\$05.00 on the wind scale (i.e., $\mathcal{O}(1000 \mathrm{~km}))$. However the oceanic upper layers are not uniform on such large scales. Oceans are indeed crowded in many regions with a large number of strongly interacting mesoscale eddies and related submesoscale filaments with scales ranging from $100 \mathrm{~km}$ to $10 \mathrm{~km}$ [Hurlburt and Hogan, 2000]. Kunze [1985] showed that the relative vorticity of these structures significantly affects the dynamics of the inertial motions and causes them to be rapidly expelled from cyclonic structures and trapped within anticyclonic ones. These eddy effects may inhibit the potential ringing of the ocean. On the other hand windforced inertial motions may affect the eddy field itself and therefore the general circulation. Such arguments emphasize the need to assess the ringing of the ocean in presence of mesoscale eddies.

[4] In this study, we make use of meteorological data sampled every 3 hours for 540 days (years 1963-1964) on the weathership "KILO" located in the North-Atlantic $\left(45^{\circ} \mathrm{N}, 16^{\circ} \mathrm{W}\right)$ to calculate the windstress, turbulent heat fluxes and solar and infrared radiative fluxes from classical formula [Large and Crawford, 1995]. No freshwater forcing is considered since salinity is assumed constant. In the next section these fluxes are used in a 1-D Mellor and Yamada. [1982] level 2.5 model (hereafter MY model) to determine which wind fluctuations are needed to trigger the resonance in the absence of eddies. Then we examine the oceanic response to the HF winds when eddies are present using a shallow water model. Results on the eddy effects are discussed in the third section. Conclusion and discussion are offered in the last section.

\section{Forcing of the Ringing Without Eddies}

[5] The impact of the HF winds has been evaluated by running simulations using the 1-D MY model and the 3 hourly meteorological data described previously. Coriolis parameter is $f=10^{-4} \mathrm{~s}^{-1}$ (corresponding to $\approx 18$ hours). Figures 1 a and $1 \mathrm{~b}$ show the time evolution of $u^{* 3}=|\tau|^{\frac{3}{2}}$ and of the wind energy flux (i.e., $\tau$.u, with $\tau$ the windstress vector and $\mathbf{u}$ the surface velocity vector). Comparison of Figures $1 \mathrm{a}$ and $1 \mathrm{~b}$ indicates that not all strong wind events produce a positive wind energy flux and therefore increase the kinetic energy: a non negligible number of strong wind events do not increase but eventually decrease the kinetic energy, which illustrates the importance of the phase relationship between the windstress direction and the surface velocity vector. As found by previous studies [Large and Crawford, 1995], not only the duration (with respect to the Coriolis period) but also the rotation of the HF wind fluctuations are important for the resonance 
a

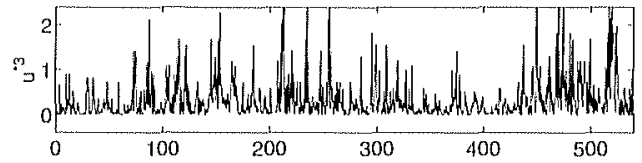

b

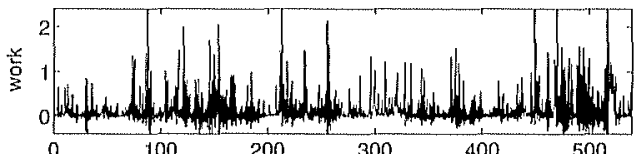

c

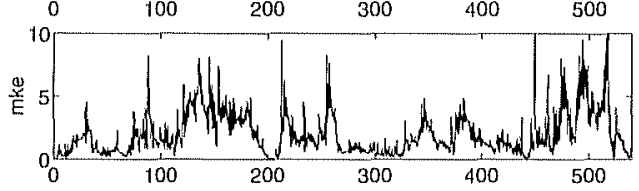

Figure 1. Results of a 3-hourly simulation: (a) $u^{*^{3}}=|\tau|^{\frac{3}{2}}$ (in $\mathrm{m}^{3} \cdot \mathrm{s}^{-3} \times 1.5 .10^{5}$ ), (b) wind energy flux ([ $\left.\tau, \mathrm{u}\right]$ (in $\left.\mathrm{m}^{3} \mathrm{~s}^{-3} \times 10^{4}\right)$ ), (c) inertial energy integrated over the water column (in $\mathrm{m}^{3} \mathrm{~s}^{-2}$ ). Abscissa units are days.

mechanism. This has been revealed by another simulation identical to the first one but with the Coriolis parameter having the opposite sign: although the magnitude of the kinetic energy averaged over 540 days is about the same, its time evolution (not shown) displays strong differences. Such result confirms that periods of large kinetic energy are present not only because of strong winds but also because these winds force the resonance. Then, to better quantify the impact of the HF wind fluctuations on the ocean ringing, we have compared the first simulation with others identical to the first one but that use the windstress averaged over 6,12 and 24 hours. The impact is clearly revealed by Table 1: when the windstress is averaged over 6, 12 and 24 hours, the total kinetic energy is decreased respectively by $1.5,3$ and 7 ! These results confirm the necessity to use a 3-hourly wind time series to force the ringing of the ocean at least, in the absence of mesoscale eddy motions.

\section{Forcing of the Ringing With Eddies}

\subsection{Simulations}

[6] The impact of mesoscale eddies on the inertial motions forced by HF winds has been evaluated by running simulations of 18-day duration. Ten windstress time series of 18-days each have been selected to correspond to periods (pinpointed with the 1-D model) where large kinetic energy persists for several days (which indicates the forcing of the resonance by the windstress). For each period two simulations, one with eddies and one without eddies, have been performed (see Figure 2). The mesoscale eddy field used is generated by the Hua and Haidvogel [1986] quasigeostrophic model and is described in Klein et al. [2004]. It is assumed barotropic and evolves with time. The resulting vorticity field (Figure $3 a$ ) is characterized by

Table 1. Statistics Over 540 Days of the Inertial Energy Integrated Over the Water Column (in $\mathrm{m}^{3} \mathrm{~s}^{-2}$ ) Obtained With the 1-D Model

\begin{tabular}{ccc}
\hline Simulations & Mean Inertial Energy & RMS Inertial Energy \\
\hline 3 hourly winds & 2.03 & 1.70 \\
6 hourly winds & 1.65 & 1.38 \\
12 hourly winds & 0.62 & 0.56 \\
24 hourly winds & 0.28 & 0.28 \\
\hline
\end{tabular}

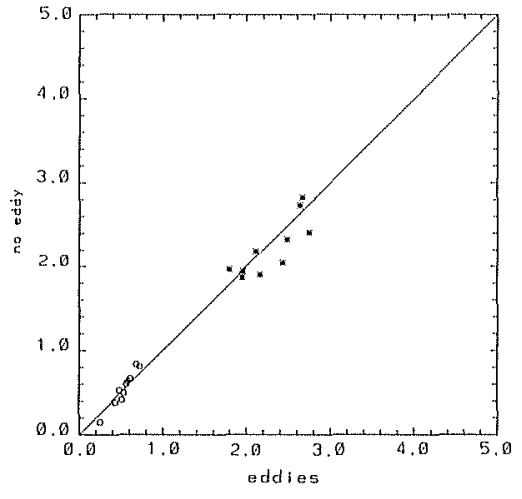

Figure 2. Scatterplot of the total inertial energy $\left(\mathrm{m}^{3} \mathrm{~s}^{-2}\right)$ at the end of the 18-day simulations. Symbols * and $o$ correspond to simulations run with respectively a 3 hourly and a 12 hourly windstress.

small-scale coherent vortices and strong vorticity fronts. Its RMS value is close to $0.05 f$. Its spectrum (not shown) has a peak at $k_{0}=5(k=1$ corresponds to the length of the domain, i.e., $1000 \mathrm{~km}$ ). The eddy field affects the dynamics of the inertial motions but there is no feedback of these motions on the eddies. The resulting model used is, as in Klein et al. [2004], a shallow water model with one active upper layer and a lower layer without motions. Equations are

$$
\begin{aligned}
& \frac{D u}{D t}+u \frac{\partial U}{\partial x}+v \frac{\partial U}{\partial y}-f v=\frac{T_{x}}{h}-g^{\prime} \frac{\partial h}{\partial x}-r(u-\bar{u}) \\
& \frac{D v}{D t}+u \frac{\partial V}{\partial x}+v \frac{\partial V}{\partial y}+f u=\frac{\tau_{y}}{h}-g^{\prime} \frac{\partial h}{\partial y}-r(v-\bar{v})
\end{aligned}
$$

$$
\frac{D h}{D t}+h\left(\frac{\partial u}{\partial x}+\frac{\partial v}{\partial y}\right)=0
$$

where $\frac{D_{.}}{D t}=\frac{\partial}{\partial t}+(u+U) \frac{\partial}{\partial x}+(v+V) \frac{\partial}{\partial v}$, with $(u, v)$ and $(U, V)$ the eastward and northward velocity components of respectively the inertial motions and the mesoscale eddy field. $h$ is the mixed-layer depth. $\left(\tau_{x}, \tau_{y}\right)$ are the eastward and northward windstress components uniform over the whole domain. The chosen values of $g^{\prime}$ (the reduced gravity constant) and $h_{o}$ (the initial mixed-layer depth) correspond to a Rossby radius $\left(r_{d}=\sqrt{g^{\prime} h_{o}} / f\right)$ equal to $6 \mathrm{~km}$. As in
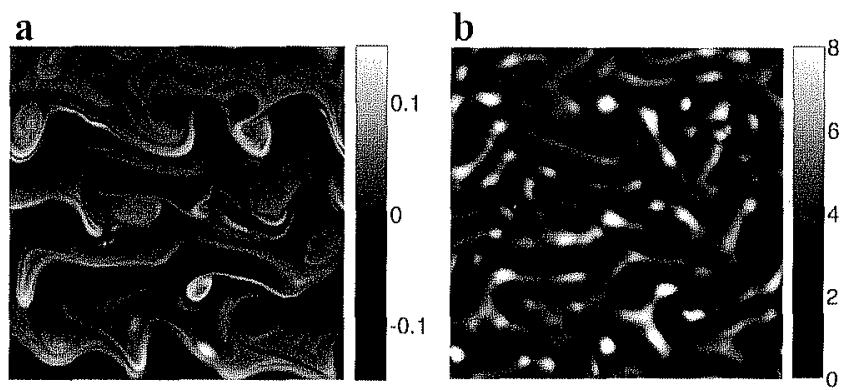

Figure 3. Characteristic snapshots of (a) the eddy vorticity scaled by $f$ and (b) inertial energy in $\mathrm{m}^{3} \mathrm{~s}^{-2}$. 
Watanabe and Hibiya [2002], we have introduced a damping rate of the inertial motions, $r=(5 \text { days })^{-1}$, to represent their vertical propagation and thus their decay in the surface layers. Overbar denotes the average over the whole domain.

\subsection{Results}

[7] A first noticeable result displayed by Figure 2 is that on a global scale, the total inertial energy $\left(\left\langle h\left(u^{2}+v^{2}\right) / 2\right\rangle\right)$ forced by the HF winds is not significantly affected by the eddies: the presence of eddies increases by 10 to $20 \%$ in some simulations the total inertial energy but in others it decreases the energy by a similar amount. When the HF winds are filtered over a 12-hourly period, the total inertial energy is significantly reduced by a factor between 4 and 5 , whether the eddies are present or not (Figure 2). This result indicates that at least a 3 hourly windstress is also required to get the correct magnitude of the total inertial energy when eddies are present.

[8] However the distribution in physical space of the wind energy input reveals the strong impact of the mesoscale eddies. This field (not shown) displays a significant spatial variability with an RMS value usually much larger than its mean value. To better understand the relation between the wind energy input and the eddy properties, we have calculated a particular index: it is the correlation between the spatial spectral components of the eddy vorticity and those of $\tau$.u (Figure 4a). This index can also be thought of as the correlation in physical space between vorticity and $\tau$.u band-passed around wavenumbers. Calculations of this correlation index have been done for each daily snapshots taken from the ten simulations when the total inertial energy is larger than $1.5 \mathrm{~m}^{3} \mathrm{~s}^{-2}$. This corresponds to 110 days. The mean value of the correlation between the eddy vorticity and $\boldsymbol{\tau}$.u (solid line in Figure 4a) is slightly negative. However when one averages only daily snapshots with positive (negative) correlations for all $k<10$, which corresponds to the dashed (respectively dotted-dashed) curve, the mean value of the correlations attains \pm 0.8 for the wavenumber close to $k=4$ and quickly collapses to the solid line of the overall correlations for the wavenumbers $k>8$. The value $k=4$ is actually quite close to the peak $(k=5)$ of the vorticity spectrum. This indicates that, for each snapshot, the wind energy input is systematically well correlated or anticorrelated with the large-scale part of the vorticity field. The explanation is that the oceanic upper layer is not just composed of one oscillator with frequency $f$ as it is with no eddies. Rather it is composed of many local oscillators with frequency $f+\zeta$ (with $\zeta$ the eddy vorticity), each one associated with an eddy. Then depending on whether the dominant frequency of the wind pulse is slightly smaller or larger than $f$, ringing will be forced in either anticyclonic eddies or cyclonic ones. Thus the HF winds "illuminate" either the anticyclonic or the cyclonic structures with the resulting wind energy input positive in the vorticity structures that are "illuminated" and negative in those of opposite sign. One consequence is that the presence of the eddies locally shifts and generally broadens the frequency band of the windstress that can force the ringing of the ocean with the result that the wind energy is injected to the ocean at much smaller scales (the scales of the local oscillators) than the wind scales. A second

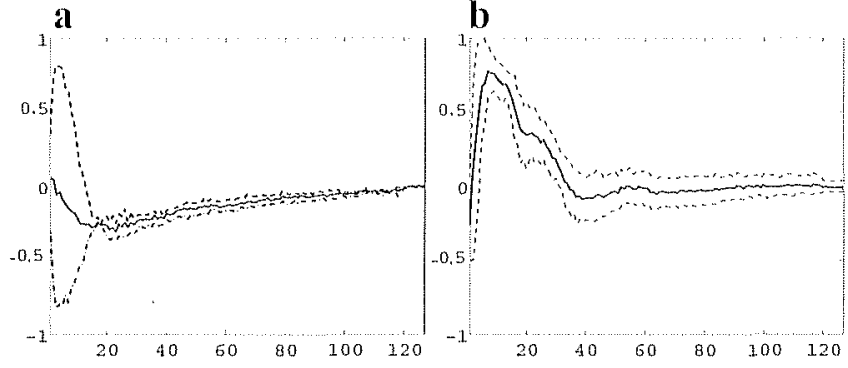

Figure 4. Correlation coefficient between spectral coefficients averaged along circular rings in wavenumber space (a) of the wind energy flux ( $\tau . \mathrm{u})$ and the eddy vorticity and (b) of inertial energy and the Laplacian of the eddy vorticity. 110 snapshots have been used. Abscissa are the wavenumbers. The different curves are discussed in the text.

consequence is that the wind-forced inertial motions are not purely inertial but are near-inertial.

[9] Another noticeable result is that the inertial energy displays a significant spatial variability (Figure 3b). To characterize this spatial variability, we have calculated the correlation between the spatial spectral components of the Laplacian of the vorticity and those of the inertial energy. The physics behind this choice is that, as explained in Klein et al. [2004], the eddy vorticity modulates the phase of the inertial motions, which make them to rapidly become spatially heterogeneous. Since the concentration of these motions is proportional to the divergence of their dispersion (that itself depends on their spatial derivatives) then the inertial energy should be linked to the Laplacian of the vorticity. Figure $4 b$ reveals that the inertial energy is positively correlated with the Laplacian of the eddy vorticity: the correlation is larger than 0.7 for the wavenumbers $5<k<14$ whereas the standard deviation (dashed lines in Figure 4b) is small. Regions of positive Laplacian vorticity where the inertial energy is large correspond to small-scale anticyclonic structures. The analytical solution proposed by Klein et al. [2004] is:

$$
\frac{\mathcal{R}^{\prime}}{\overline{\mathcal{R}}}=\frac{t l_{i e}^{2}}{8} \nabla^{2} \zeta_{T}
$$

with $\mathcal{R}=\mathcal{R}^{\prime}+\overline{\mathcal{R}} . \mathcal{R}^{2}$ is the inertial energy. $\nabla^{2} \zeta_{T}$ is the Laplacian vorticity field truncated so as to retain only the scales larger than $l_{i e}$ (with $l_{i e}^{2}=2 \pi f i_{t}^{2} t$ ). We have found that the upper value $k=14$ displayed by Figure $4 \mathrm{~b}$ and the RMS value of the inertial energy (about half its mean value) well match these analytical expressions when a time of $t \approx$ 3.3 days is used: such time scale leads to $l_{i e}=70 \mathrm{~km}$ (which corresponds to $k=14$ ) and $\mathcal{R}^{\prime} / \overline{\mathcal{R}} \approx 0.7$. This result expresses an equilibrium between the wind forcing and the spatial redistribution by the eddies. The time scale of 3.3 days appears to be robust but the mechanisms that set up this valuc have still to be investigated. They certainly involve the HF wind variability since a simulation (not shown) run with a windstress averaged over 6 days leads to a noisy inertial energy field uncorrelated with the eddy field. They may also involve the eddy characteristics since the enstrophy time scale is also close to 3 days. These results 
indicate however that, with a real wind stress, the inertial motions are efficiently and permanently concentrated by the eddy field in quite specific small-scale anticyclonic structures.

\section{Conclusions}

[10] Results of this study indicate that a turbulent eddy field does not inhibit the impact of HF winds on the inertial motions. A three-hourly wind time series is at least required to stimulate the ringing of the ocean both with and without eddies. However the eddies efficiently disperse and aggregate inertial motions such that large inertial energy is principally found in small-scale anticyclonic structures. As a consequence any vertical mixing induced by the inertial energy is heterogeneous and strongly related to the eddy field properties. Our study does not consider any feedback of this mixing on the eddies. But previous studies indicate that such heterogeneous mixing may in turn affect the nonlinear interactions between the eddies and consequently the eddy field itself. Klein et al. [2003] for example showed that such heterogeneous mixing breaks the cyclone-anticyclone symmetry preexisting in an eddy field at a time scale much smaller than the mixing time scale. Then, within the context of the recent findings on the impact of $\mathrm{HF}$ winds on the general oceanic circulation [Wunsch and Ferrari, 2004], the emerging picture is that this general circulation could be modified by the HF wind energy input, not directly by the small-scale mixing induced by the inertial energy, but indirectly through the mesoscale eddies.

[11] More detailed studies on the organization of the wind-forced inertial energy by an eddy field, and in particular on the resulting heterogeneous mixing, are needed. Eddies with an $\mathcal{O}(1)$ Rossby number may enlarge the potential ringing of the ocean and in that case the feedback effects of the resonance on the eddies should be explicitly taken into account. Two other mechanisms not considered in our study should be investigated: the vertical propagation of the inertial motions in the deeper layers and the vertical velocity induced by the eddy field. Previous results indicate that the inertial energy injected downward does not go far into the deeper layers since it is usually trapped within the seasonal thermocline [Wang, 1991; Klein and Tréguier, 1995]) and is in phase with the maxima of the inertial energy in the upper layers. This should lessen the stratification below the mixed-layer and therefore enhance the mixedlayer deepening. The presence of the vertical velocity related to the eddy field should also be investigated since it may affect the mixed-layer deepening in those regions.

[12] Acknowledgments. This work is supported by NCAR (USA) and the CNRS and IFREMER (FRANCE). Some of the calculations reported here were done on the NEC SX5 of the IDRIS (Orsay, France).

\section{References}

Alford, M. H. (2001), Intemal swell generation: The spatial distribution of energy flux from the wind to mixed-layer near-inertial motions, $J$. Phys. Oceanogr:, 31, 2359-2368.

Hua, B. L., and D. B. Haidvogel (1986), Numerical simulations of the vertical struchure of quasi-geostrophic turbulence, J. Almos. Sci., 43, $2923-2936$.

Hurlburt, H. E., and P. J. Hogan (2000), Impact of $1 / 8^{\circ}$ to $1 / 64^{\circ}$ resolution on Gulf Stream model-data comparisons in basin-scale subtropical Atlantic Ocean models, Dyn. Atmos. Oceans, 32, 283-329.

Klein, P., and A. M. Tréguier (1995), Dispersion of wind-induced inertial waves by a barotropic jet, J. Mar: Res., $53,1-22$.

Klein, P., B. L. Hua, and X. Carton (2003), Emergence of cyclonic structures due to the interaction between near-inertial waves and mesoscale eddies, Q. J. R. Meteorol. Soc., 129, 1-13.

Klein, P., S. Llewellyn-Smith, and G. Lapeyre (2004), Spatial organisation of inertial energy by an eddy field, $Q . J . R$. Meteorol. Soc., 130, 11531.166.

Kunze, E. (1985), Near-inertial wave propagation in geostrophic shear, J. Phys. Oceanogr:, 15, 544-565.

Large, W. G., and G. B. Crawford (1995), Observations and simulations of upper-ocean response to wind events during the ocean storms experiment, J. Phys. Oceanogr:, 25, 2831-2852.

Mellor, G. L., and T. Yamada (1982), Development of a turbulence closure model for geophysical fluid problems, Rev. Geophys. Space Phys., 20, $851-875$.

Skillingstadt, E. D., W. D. Smyth, and G. B. Crawford (2000), Resonant wind-driven mixing in the ocean boundary layer, $J$. Phys. Oceanogr:, 30, $1866-1890$.

Wang, D. P. (1991), Generation and propagation of inertial waves in the subtropical front, J. Mar: Res., 49, 619-633.

Watanabe, M., and T. Hibiya (2002), Global estimates of the wind-induced energy flux to inertial motions in the surface mixed layer, Geophys. Res. Lett., 29(8), 1239, doi:10.1029/2001GL014422.

Wunsch, C., and R. Ferrari (2004), Vertical mixing, energy and the general circulation of the ocean, Anmu. Rev. Fluid Mech., 36, 281-314.

P. Klein and G. Lapeyre, Laboratoire de Physique des Océans, IFREMER, Plouzané, France. (pklein@ifremer.fi)

W. G. Large, Climate and Global Dynamics Division, NCAR, Boulder; CO, USA 